\title{
Development and evaluation of an online grading system for pinto beans using machine vision and artificial neural network
}

\section{Mahmoud Omid* and Aghil Salehi}

Department of Mechanics of Agricultural Machinery, Faculty of Agricultural Engineering and Technology, University of Tehran,

Karaj, Iran

Email: omid@ut.ac.ir

Email: Salehi@ut.ac.ir

*Corresponding author

\section{Mahdi Rashvand}

Machine Design and Mechatronics Department, Institute of Mechanics, Iranian Research Organization for Science and Technology, Tehran, Iran

Email: Mahdi.rashvand@irost.ir

\section{Mahmoud Soltani Firouz}

Department of Mechanics of Agricultural Machinery, Faculty of Agricultural Engineering and Technology, University of Tehran,

Karaj, Iran

Email: mahsoltani@ut.ac.ir

\begin{abstract}
The design of an intelligent system for qualitative evaluation of beans product impurities is the most important step necessary to make a bean sorting machine. In this research, a real-time system of pinto beans sorting (from red, white, and damaged beans, and stones) was designed and developed by combining image processing and artificial neural networks (ANNs). In total, six parameters were selected from the statistical characteristics of beans for classification of pinto beans from other beans and stones. Several ANN classifiers each with different number of neurons in the hidden layer were trained to determine the optimal structure. Optimal topology of ANN classifier was 6-12-8-2. In the first step the offline system was evaluated. The correct classification rate for pinto, white, red, and damaged beans and stones were $86.27,100,100,54.9$ and $65.3 \%$, respectively. The average accuracy of offline method was $81.2 \%$. The corresponding MSE were calculated as $0.05,0.059$, $0.013,0.099$ and 0.096 , respectively. The accuracy of the online sorting system for pinto bean from others was $97.87 \%$. The results showed that the designed system combined with ANN technique had acceptable efficiency in pinto been grading.
\end{abstract}

Keywords: artificial neural networks; ANNs; classification; image processing; pinto beans. 
Reference to this paper should be made as follows: Omid, M., Salehi, A., Rashvand, M. and Firouz, M.S. (2020) 'Development and evaluation of an online grading system for pinto beans using machine vision and artificial neural network', Int. J. Postharvest Technology and Innovation, Vol. 7, No. 1, pp.1-14.

Biographical notes: Mahmoud Omid received his BEng in 1989 from the Newcastle University, UK, and his MS and $\mathrm{PhD}$ in Telecommunications from the University of Electro-Communications, Japan, in 1994 and 1997, respectively. He is currently a Full Professor in the Faculty of Agricultural Engineering and Technology at the University of Tehran (UT), Iran. His special field of interest include artificial neural networks, fuzzy logic, machine vision, control and automation. He was authored and co-authored more than 300 articles in international journals and conferences. His current research interests include computational intelligence and machine vision in the areas of agriculture and biosystems engineering as well as agri-food industries.

Aghil Salehi is graduated from the Tehran University in Agriculture Machinery and MSc in the field of Postharvest Technology. Also he is interested in food engineering and agriculture machinery.

Mahdi Rashvand is graduated of MSc from the Tehran University and his field was Postharvest Technology. He is currently a $\mathrm{PhD}$ student at the Machine Design and Mechatronics Department, Institute of Mechanics, Iranian Research Organization for Science and Technology. His expert is food technology and machine learning.

Mahmoud Soltani Firouz is an Assistant Professor in Department of Mechanic of Agricultural Machinery, Faculty of Agricultural Engineering and Technology, University of Tehran, Karaj, Iran. His research area is food engineering.

\section{Introduction}

Grains are one of the most important sources of protein and energy for humans and have a special position in nutrition and economics (Tadesse et al., 2015). Beans contain $25-20 \%$ protein and $56-56 \%$ carbohydrates, and are used in many developing countries as one of the most important sources of plant proteins (Jiang et al., 2014). The grading of products is of great importance (Ercisli et al., 2012). As the main reason for the non-acceptance of domestic products in overseas markets is the low quality of the products in terms of their incongruity (Piotr, 2011). The weak classification and sorting methodology has caused a reduction of exported product. Therefore, design and developing an automatic pinto beans sorting system based on machine vision can improve the quality of the product, abolish inconsistent manual evaluation, and reduce dependence on available manpower.

Agricultural production is subjected to the rating of various types of fruits and other agricultural products based on size, colour, appearance and other factors, and the classification of impurities, fruits and corrosive products (Kaliramesh et al., 2013). Qualitative evaluation and product sorting in terms of colour are two important factors that are used to remove defective units and to increase the product marketability of 
products. Therefore, the design of an automated intelligent system for real-time evaluation and separation of beans product impurities seems to be the first step in making the bean grading machine (Arboleda et al., 2018).

In some cases, due to the small size of the farm and the use of traditional tools, the seeds of the beans are mixed together (Kuo et al., 2014). For example, pinto beans contain a small percentage of red and white beans. Eventually, the beans are harvested by a cereal combine. So that the beans are first arranged manually and rows on the ground to dry. After drying the crop, the combine enters the ground, and the workers manually pour beans into the combine, which causes the stones and other waste to enter the seed of beans. The main purpose of this study is to identify and isolate pinto beans from rocks, frozen beans, red beans and white beans.

The grading of agricultural products using the visual machine has long been the subject of many researches (De Oliveira et al., 2016). In recent years, computer-generated artificial classifiers that are intended to mimic human decision making for product quality have been studied intensively. Hong et al. (2002) developed an algorithm for rice grading in terms of appearance, including cracking, fracturing, whitening, and also whether the seeds were separated or grouped. He was able to accurately measure the operation with an accuracy of more than $98.8 \%$. Lorestani et al. (2006) designed and evaluated a fuzzy logic system (FIS) for grading golden delicious apples based on colour and size. In this system, an image captured by the camera is sent to the computer to be automatically extracted. This system showed the detection accuracy of $90.8 \%$. Chen et al. (2010) used a fuzzy analysis and ANN in order to classify five varieties of Chinese corn. Their images were obtained by a scanner, and a total of 58 features of each variety, including 30 morphological features and 28 colour attributes, were obtained. Their experiments showed that the average classification accuracy for the five varieties of corn was more than $90 \%$.

The combination of computer vision and artificial neural networks (ANNs) is similar to the coordinated activity of human brain and eyes. Omid et al. (2010) used a combination of the ANN and principal component analysis (PCA) techniques with the help of acoustic effect for sorting of open and closed pistachios. Mollazade et al. (2012) used three techniques including ANN, support vector machines (SVMs) and decision trees, to classify raisins. They divided raisins into four groups (green, blubber green and black). They concluded that the ANN classifier with 4-6-7 structure had the highest precision. Teimouri et al. (2016) used image processing and ANN to classify almonds into five different classes. For this purpose, 215 shape, colour, and texture features were extracted from the images of almonds. The number of the inputs in the feature vector were reduced from 215 to 18 by using sensitivity analysis (SA) and PCA (Teimouri et al., 2016).

The harvested beans product usually has impurities that lead to reduced quality and dissatisfaction the customer; Like weed and sand. The manually cleanup of these material are time-consuming and also cost of labor increase, which is not economic. Therefore, providing an automatic method for separating intact pinto beans is essential. The purpose of this study is to develop an automated system that can detect pinto beans in terms of colour, size, deterioration and other waste materials using the combined technology of machine vision, image processing and ANN classifier. 


\section{Material and methods}

\subsection{Bean sorting machine}

Colour and size are the most important features for a fast and accurate grading of bulk beans. Therefore, a machine vision system for sorting beans by colour and size features was designed and implemented. The schematic of the prototype sorter is shown in Figure 1. The hardware included a conveyer belt $(80 \mathrm{~cm}$ in length and $25 \mathrm{~cm}$ in width), an induction motor (MotoGen: Iran, $0.37 \mathrm{~kW}, 380 \mathrm{~V}, 2.1 \mathrm{~A}$ ), a black box (lighting chamber), light sources, a video camera (PROLINE-565s, 480TVlines), a capture card (MINI USB TV BOX from ALFEX) and a PC. The conveyor belt was used to transport beans for processing. A green colour was used as a background for image processing. As seen in Figure 1, beans are discharged on the belt at the entrance of the conveyer belt. The raisins pass below the lighting chamber, located $27 \mathrm{~cm}$ away from the entrance. The camera mounted above the chamber, captures the images of the beans as they pass and transfers the data to the capture card via a cable link to PC for storage and postprocessing analysis.

The pneumatic section of the sorting machine consists of a compressor with $15 \mathrm{~L}$ tank and a maximum pressure of $6 \mathrm{bar}$, an air diffuser, eight $24 \mathrm{~V}$ two-channel/one-way solenoid valves with $6-8$ bar working pressure and a few no. 8 hoses and fitters. The air diffuser is responsible for distributing the compressed air among valves. Fitters $(1 / 4-1 / 8$ and 1/4-1/4 types) are used to connect the valves to the nozzles and the compressor via hoses in different parts of the sorting machine. These fitters have been used as air nozzles as well. To control the valves, an electronic circuit based on an AVR micro-controller was used. The microcontroller is in charge of receiving the code number of the active pneumatic valves from the PC and opening them for the necessary time to eliminate unsuitable raisins. The proper time to keep the circuit closed was found experimentally as $75 \mathrm{~ms}$. The microcontroller receives the data via a computer serial RS232 port. To convert the voltage level while transferring information with the PC, a MAX232 chip was used. The information transfer rate was set as 9,600 Bit/s. Hence, by using an $11,059,200 \mathrm{~Hz}$ crystal, transferring information could be carried out perfectly because this frequency is an integer multiple of the speed rate $(9,600)$. The A port of the microcontroller is defined as the exit and its 34 to 40 pins are connected to the 1 to 7 valves, respectively. To open each of the valves, the related pin on the micro-controller is configured and the valve stays open as long as the timer has been programmed. Since the microcontroller cannot open the valves on its own, the ULN2003 chip was used to convert voltage and open the $24 \mathrm{~V}$ valves.

After extracting the features by the computer, these characteristics are converted to signals and commands are issued to solenoid valves. In the present study, these characteristics are the recognition of the colour and good shape and the location of the product on the conveyor belt. The electronic and control section of the sorter consisted of Atmega16 microcontroller board and needed circuitry to control the pneumatic part and also to receive the controlling commands from the computer. The main task of this part is carried out by an AVR microcontroller made by ATMEL. The microcontroller receives the data via a computer serial RS232 port. To convert the voltage level while transferring information with the computer, a MAX232 chip was used. 
Figure 1 Schematic of the system for sorting pinto beans (see online version for colours)

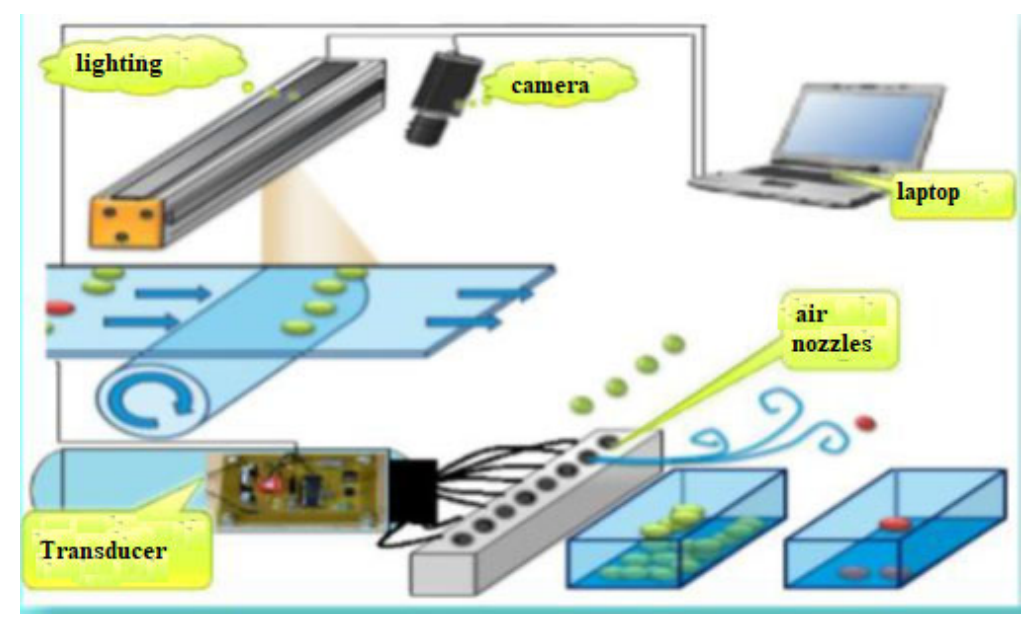

In the present study, colour of the beans is one of the main characteristics that should be identified by the designed algorithm (Figure 2). All the colour spaces (RGB, HSI, etc.) are three-dimensional and are represented by three axes perpendicular to each other. In RGB colour space, for instance, they are shown in red, green, and blue. The dispersion of values of image pixels in the RGB colour space forms a centralised area along the axes [Figure 3(a)]. This type of distribution cannot give a simple min-max limits as in thresholding, which is a simple, yet effective, way of partitioning an image into a foreground and background. But the values of the same pixels in the HSI colour space are distributed at a wider range [Figure 2(b)]. Hence, in this research the HSI space was used.

Figure 2 Diagram of process of developed system

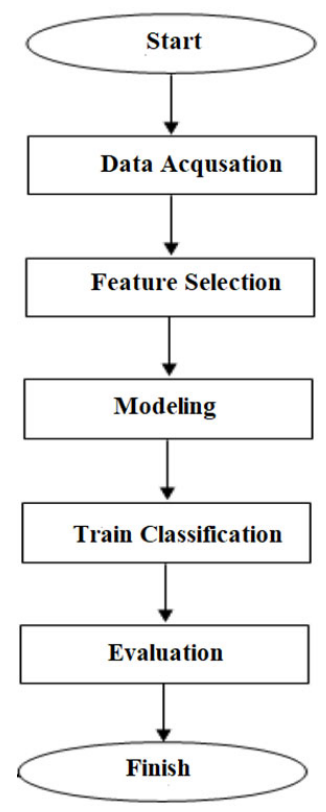


Figure 3 Spread of the image pixel values in, (a) RGB colour space (b) HSI colour space (see online version for colours)

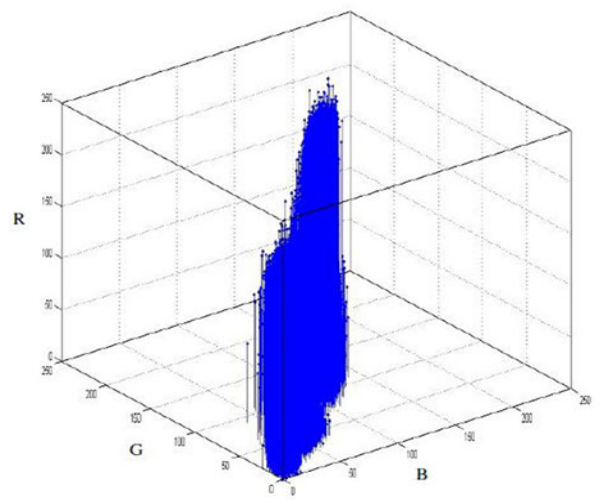

(a)

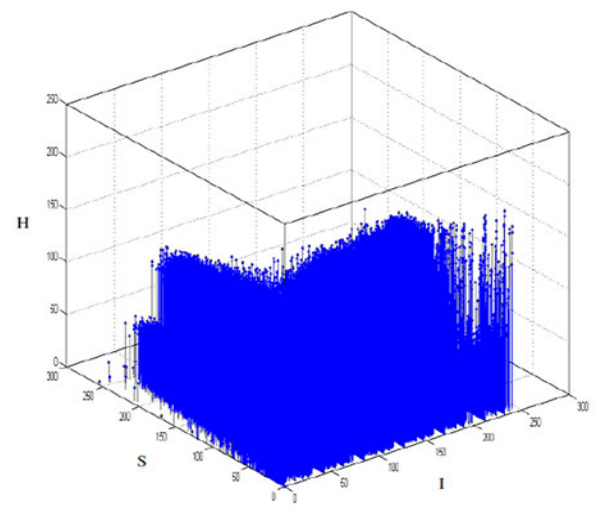

(b)

\subsection{Background elimination}

After image acquisition, the background must be eliminated by removing the image of the product. In general, the background colour must be different to the colour of the beans to remove the background. There are several different methods for eliminating the background. Here, a mask was designed to remove the background and the HSI colour space was used to apply this technique. Initially, the components of the RGB of image were extracted and are converted into HSI components. Subsequently, by subtracting G-component from I-component, an image was obtained. In the final step, using a conditional if statement, the value of the image background was set to zero and the beans were given a value of 1 (Figure 4).

Figure 4 Background elimination, (a) original image (b) separated background image (see online version for colours)

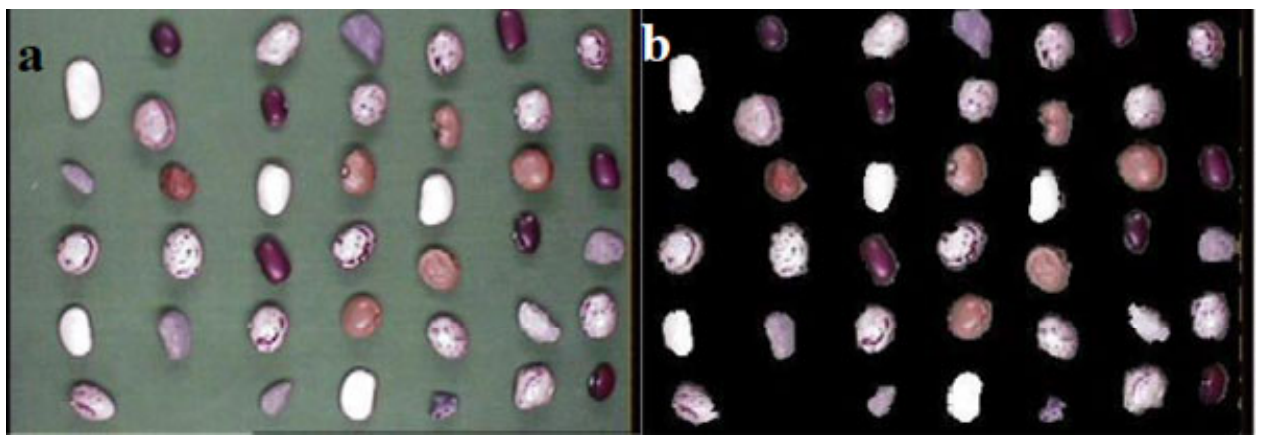

\subsection{Segmentation and classification}

HSI images were used to identify beans. The pixel values of red bean (in RGB colour space) are close to zero as a result of their darkness [Figure 5(a)] and the pixel values for white beans are close to 255 as a result of their brightness [Figure 5(b)]. The brightness 
for pinto beans is due to the presence of white and red colours and lies between white beans and red beans. Therefore, statistical techniques (mean, variance, skewness, range, and kurtosis) can be used on pixels to identify various beans (Table 1).

Figure 5 (a) Brightness for component R colour space of red bean (b) Brightness for component $\mathrm{R}$ colour space of white bean (see online version for colours)

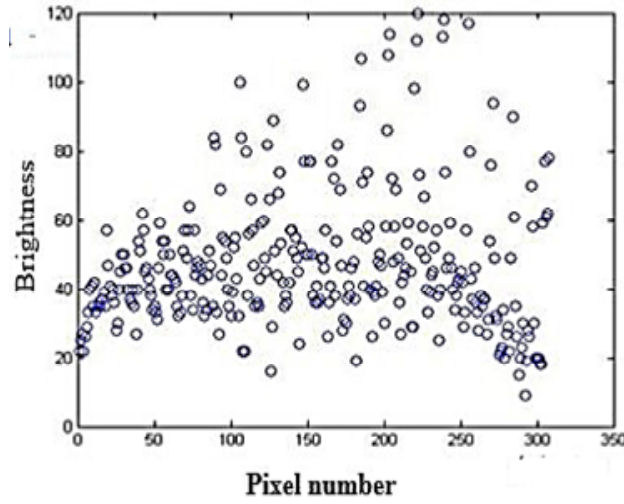

(a)

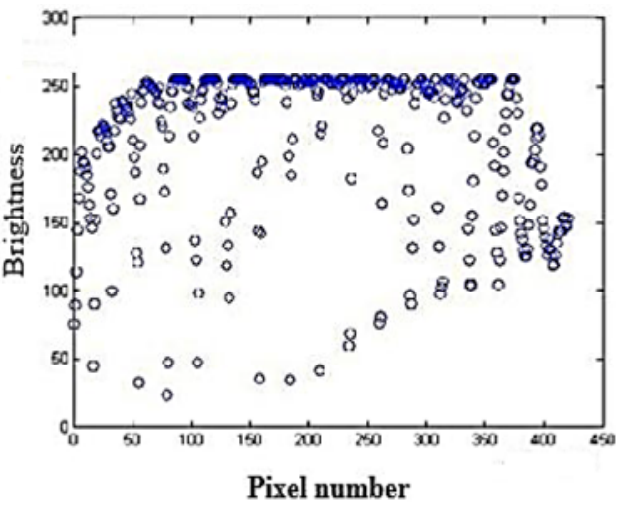

(b)

\section{$2.4 A N N S$}

ANNs are one of the subcategories of artificial intelligence that are widely used in data analysis and modeling (Soltani et al., 2015). Various types of ANNs have been developed. Multilayer perceptron (MLP) is commonly used for classification and function approximation purposes (Almaliki et al., 2016). Input layer, hidden layer(s) and output layer constitute the MLP structure. For development of ANN models, two hidden layers with hyperbolic tangent activation functions were used. To avoid over training, the ANN that often results in improper model learning, data were divided into three parts: $60 \%$ of the data for training, $15 \%$ of the data was used for cross validation, and $25 \%$ for testing.

\subsubsection{Offline classification of beans}

After the beans were pulled down by distributor and placed on each row in front of a pneumatic valve, the camera begins to take photos and detects undesirable beans by following the steps that was mentioned before. After detecting undesirable bean, its coordinates should be found and send the related code to the microcontroller, so that the pneumatic valve corresponds to that point. To separate beans in the input layer, six statistical parameters (Table 1) were fed to ANN, that each of which is related to a variable or feature, as inputs (Figure 6). The hidden layers also contain several neurons. The output layer was based on a variety of beans including five neurons, but this model was only implemented in the NeuroSolutions software in offline mode. The output values contain a row vector with five members including 0 and 1 are introduced to the network. 
Table 1 The used statistical indexes for features extraction

\begin{tabular}{lc}
\hline Feature & Formula* $^{*}$ \\
\hline Mean & $\frac{1}{n} \sum_{i=1}^{n} x_{i}$ \\
Variance & $\frac{1}{n} \sum_{i=1}^{n}\left(x_{i}-\mu\right)$ \\
Skewness & $\frac{1}{n} \sum_{i=1}^{n}\left(x_{i}-\mu\right)^{3}$ \\
Range & $\max \left(x_{i}\right)-\min \left(x_{i}\right) \quad i=1: n$ \\
Kurtosis & $\frac{1}{n} \sum_{i=1}^{n}\left(x_{i}-\mu\right)^{4}$ \\
\end{tabular}

Notes: *in this table, $n, x, \mu$ are the number of sample pixels in the image, the value of the pixels and mean, respectively.

Figure 6 The ANN structure for classification of pinto beans

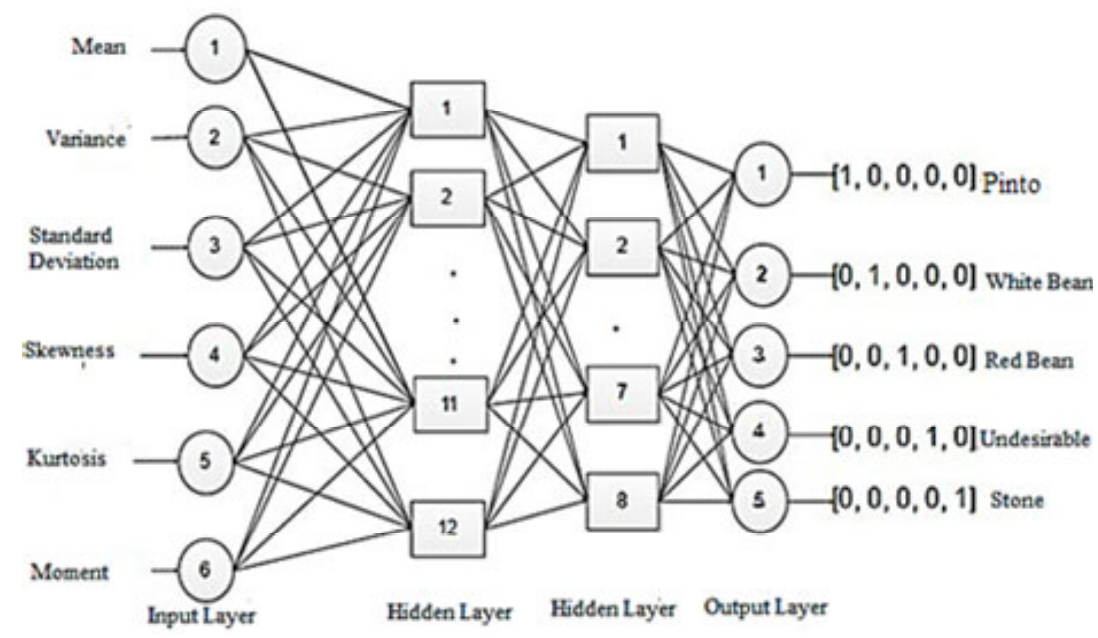

\subsubsection{Online classification using $A N N$}

MLPs are good solution for classification purposes and have been used extensively for the grading of agricultural products. In this research, MLPs have been used with back propagation learning method to grade pinto, white, red, frozen bean and stone, using the extracted features from the images that taken from the camera. Statistical characteristics for beans were considered, so the network inputs were statistical parameters and the network outputs were desirable or undesirable condition of beans. A sample group including 800 randomly selected pinto beans and 200 others was prepared, each sample was taken randomly and the statistical parameters necessary for network training were 
extracted. The selected network was implemented in MATLAB software, neural network toolbox.

In order to test the pinto bean sorting system, 400 beans were randomly selected and four experts were asked to classify the beans in small, medium and large sizes. After testing, 223 beans were identified as medium, 83 were large and 94 were identified as small beans by experts. Then the length of beans was measured by the caliper, which was obtained for average, 11.9, 9.9 and $7.5 \mathrm{~mm}$ for large, medium and small beans. The size of each pixel in this system was $0.625 \mathrm{~mm}$, the sizes obtained for large, medium and small beans are $3.7,6.18$, and 4.68 pixels, respectively. After identifying by experts, each bean group was imprinted on the device in real conditions, and three attributes of area, large diameter and small diameter were extracted for each bean and used as inputs to train the MLP net. In the hidden layer, the hyperbolic tangent was used and $60 \%$ of the data was randomly selected for training, $15 \%$ of the data for cross validation, and finally the remaining $15 \%$ of data for testing the classifier. The training process was carried out in MATLAB software, was similar to the previous section, which the result of this test is used for grading in online mode.

\subsection{Performance analysis}

The performance analysis of classification can be evaluated by the semi-global performance matrix, known as the confusion matrix (Teimouri et al., 2014). This matrix contains information about actual (desired) and predicted classification data obtained by the ANN classifier. Table 2 shows the confusion matrix for a five-class classifier which represents how the instances are distributed over actual (columns) and desired (rows) classes.

Table 2 Confusion matrix for classification of five classes

\begin{tabular}{lccc}
\hline & $C_{1}$ & $\cdots$ & $C_{5}$ \\
$C_{1}^{*}$ & $n_{11}$ & $\ldots$ & $n_{15}$ \\
$\mathrm{~N}$ & $\mathrm{~N}$ & $\mathrm{O}$ & $\mathrm{N}$ \\
$C_{5}^{*}$ & $n_{61}$ & $\ldots$ & $n_{55}$ \\
\hline
\end{tabular}

The terms $\left(n_{i j}\right)$ correspond to the pixels that are classified into class number $i$ by the ANN classifier (i.e., $C_{i}^{*}$ ), when they actually belong to class number $j$ (i.e., $C_{j}$ ). Accordingly, the right diagonal elements $(i=j)$ correspond to correctly classified instances, while off-diagonal terms $(i \neq j)$ represent incorrectly classified ones. When considering one class $i$ in particular, one may distinguish four kinds of instances: true positives (TP) and false positives (FP) are instances correctly and incorrectly identified as $C_{i}^{*}$, whereas true negatives (TN) and false negatives (FN) are instances correctly and incorrectly rejected as $C_{i}^{*}$, respectively. The corresponding counts are determined as $n_{T P}=n_{(i, i)}, n_{F P}=n_{(i,+)}$ $-n_{(i, i)}, n_{F N}=n_{(+, j)}-n_{(i, i)}$ and $n_{T N}=n-n_{T P}-n_{F P}-n_{F N}$ where $n_{(i,+)}$ and $n_{(+, j)}$ are the sums of the confusion matrix elements over row $i$ and column $j$, respectively.

The classification performances were measured based on the values of the confusion matrix, such as percentage of specificity, sensitivity, precision, accuracy, area under the curve (AUC) (Teimouri et al., 2016). To assess the accuracy and performance of ANN 
models, the correlation coefficient (R), mean absolute error (MAE) and mean square error (MSE) were used; these statistical criteria defined as equations (1) to (4).

$$
\begin{aligned}
& \text { Accuracy }=\frac{n_{T P}=n_{T N}}{n_{T P}+n_{T N}+n_{F P}+n_{F N}} \\
& R=\sqrt{\frac{\left(\sum_{i=1}^{n}\left(P_{i}-\overline{P_{i}}\right)\left(O_{i}-\overline{O_{i}}\right)\right)}{\sum_{i=1}^{n}\left(P_{i}-\overline{P_{i}}\right)^{2} \sum_{i=1}^{n}\left(O_{i}-\overline{O_{i}}\right)^{2}}} \\
& M A E=\frac{\sum_{i=1}^{n}\left|P_{i}-O_{i}\right|}{n} \\
& M S E=\frac{\sum_{i=1}^{n}\left(P_{i}-O_{i}\right)^{2}}{n}
\end{aligned}
$$

where $P_{i}$ is predicted value, $O_{i}$ is observed value, $\bar{P}_{i}$ is average value for $P_{i}, \overline{O_{i}}$ average value for $O_{i}$ and $\mathrm{n}$ is the number of observation.

\section{Results and discussion}

\subsection{Offline evaluation: classification using NeuroSolutions}

Four types of beans (pinto, white, red and damaged beans), along with stones, were used in this investigation. From each sample, 100 beans were randomly selected and taken from it. Data from statistical parameters were saved for analyse in the computer. Structure of ANNs has an important role on output of the developed models. The main parameters affecting the performance of ANNs are input variables, number of hidden layers and number of nodes in each hidden layer. So, in this research, MLP with two hidden layer was used in the structure of ANNs. To obtain the best model with the highest performance, ANNs with different number of neuron in the hidden layer were performed and evaluated. Finally, the best accuracy that obtained was reported. The ANN classifier consisted of six neurons in the input layer, two hidden layers (12 neurons in the first layer, and 8 neurons in the second layer) and five neurons in the output layer. This network was developed in NeuroSolutions software. After training and validating the network according to Tables 3 and 4, the correct classification rate for pinto, white, red, damaged and stones were $86.27,100,100,54.9$ and $65.3 \%$ respectively. The corresponding correlation coefficients (R) were $0.83,0.98,0.96,0.62$ and 0.83 respectively. Other statistical criteria such as MSE and MAE are presented in Table 4. The overall accuracy of the classifier was $81.2 \%$ for four types of beans and stone.

For the purpose of sorting of pinto beans from other undesirable substances, 400 pinto beans and 100 other items were randomly selected and introduced to the MLP network in NeuroSolutions software. The optimum network had a 6-12-8-2 structure. The performance of this network is shown in Tables 5 and 6 . The correct classification for pinto and other undesirable substances was $97 \%$ and $86.6 \%$, respectively, and the R value 
was 0.83 and 0.86 , respectively. For pinto beans and other undesirable substances, the overall accuracy of the model was $91.8 \%$.

Table 3 Confusion matrix of ANN obtained from the test dataset

\begin{tabular}{llccccc}
\hline & \multicolumn{5}{c}{ Actual } \\
\cline { 3 - 7 } & & Pinto & White & Red & Damaged & Stone \\
\hline Predicted & Pinto & 44 & 0 & 0 & 7 & 1 \\
& White & 0 & 53 & 0 & 0 & 0 \\
& Red & 0 & 0 & 46 & 2 & 1 \\
& Damaged & 3 & 0 & 0 & 28 & 15 \\
& Stone & 4 & 0 & 0 & 14 & 32 \\
\hline
\end{tabular}

Table 4 Performance of the best topology of ANN (6-12-8-5)

\begin{tabular}{lccccc}
\hline Criteria & Pinto & White & Red & Damaged & Stone \\
\hline MSE & 0.050 & 0.0059 & 0.0133 & 0.099 & 0.0965 \\
MAE & 0.116 & 0.055 & 0.0640 & 0.197 & 0.191 \\
R & 0.831 & 0.986 & 0.959 & 0.628 & 0.631 \\
Percent correct & 86.27 & 100 & 100 & 54.90 & 65.30 \\
\hline
\end{tabular}

Table 5 Confusion matrix of ANN obtained from the test dataset

\begin{tabular}{llcc}
\hline & \multicolumn{2}{c}{ Actual } \\
\cline { 3 - 4 } & & Pinto & Undesirable substances \\
\hline \multirow{2}{*}{ Predicted } & Pinto & 100 & 13 \\
& Undesirable substances & 3 & 84 \\
\hline
\end{tabular}

Table 6 Performance of the best topology of ANN (6-12-8-2)

\begin{tabular}{lcc}
\hline Criteria & Pinto & Undesired substances \\
\hline MSE & 0.0749 & 0.064 \\
MAE & 0.174 & 0.161 \\
R & 0.837 & 0.861 \\
Percent correct & 97.087 & 86.590 \\
\hline
\end{tabular}

\subsection{Online evaluation: classification using MATLAB}

The online experiments were performed using the ANN developed in MATLAB. The MLP having 12 neurons in the first hidden layer and 8 neurons in the second hidden layer performed best accuracy (Figure 7). It should be noted that all beans must be aligned in the direction of the nozzles before the fall. This is done by a distributer. The distributer was designed to guide the beans in a series of cylinders. The camera started to take photos and detected undesirable beans after the beans are pulled by the distributer and placed on each row in front of a nozzle. After detecting undesirable beans, the coordinates of its centroid is computed and the required code is send to the microcontroller so that the valve operates on that point. By detecting the desired beans, 
the program is set to zero instead. In other words, it turns into black in order to realise only undesired beans on the plane.

Figure 7 The developped MLP for sorting pinto beans in MATLAB (see online version for colours)

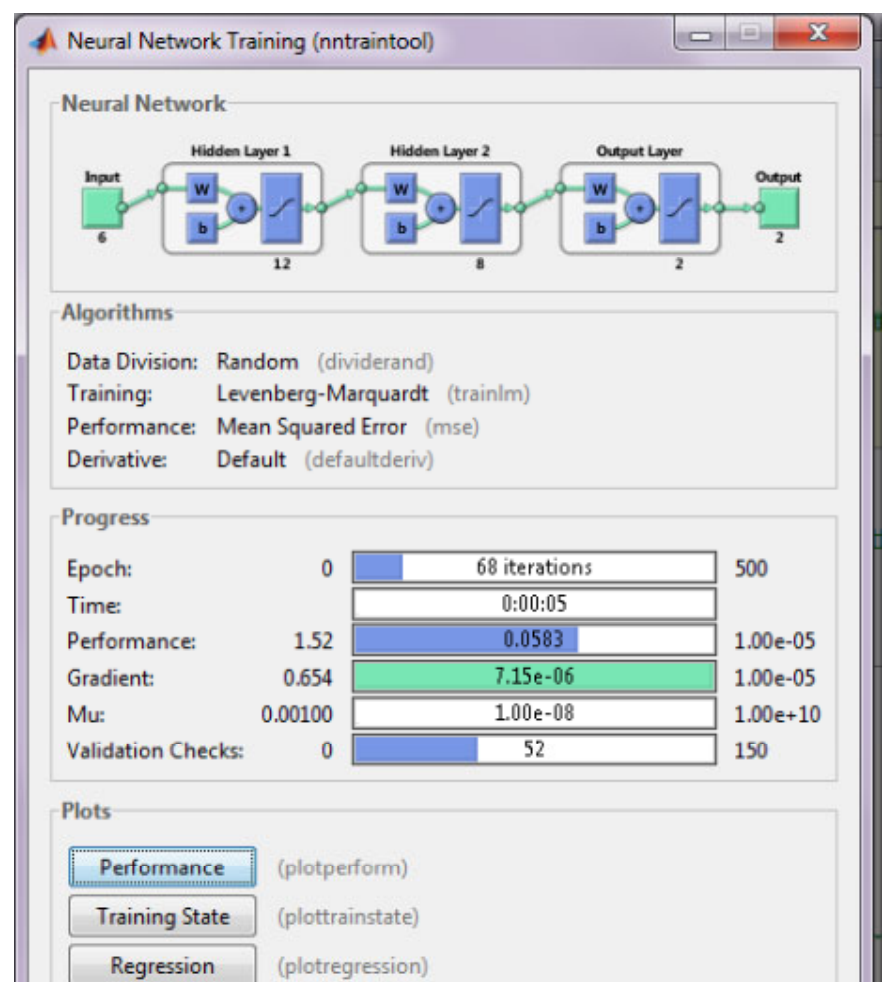

By decreasing the number of neurons, the accuracy decreased and no improvement was observed with increasing the number of neurons. The overall accuracy of network for classifying pinto beans from other materials was $98 \%$ using 6-12-8-2 topology. For this experiment, 400 beans (200 desirable and 200 undesirable) were randomly selected. In order to find the accuracy of the program in identifying the desired beans among undesirable ones. This test was repeated three times, which the results are presented in Table 6. Based on the results, the accuracy of classifier for desirable and undesirable beans for the first repetition was 97.02 and $97.97 \%$ for pinto and others, respectively.

Table 7 Confusion matrix of ANN obtained from the test dataset

\begin{tabular}{llcccc}
\hline Repetition & \multicolumn{1}{c}{ Sample } & Total & Pinto beans & Undesired substances & Accuracy \\
\hline \multirow{2}{*}{2} & Pinto beans & 202 & 196 & 6 & 97.02 \\
& Undesired substances & 198 & 4 & 194 & 97.97 \\
2 & Pinto beans & 195 & 193 & 2 & 98.97 \\
& Undesired substances & 205 & 7 & 198 & 96.58 \\
3 & Pinto beans & 196 & 193 & 3 & 98.46 \\
& Undesired substances & 204 & 7 & 197 & 96.56 \\
\hline
\end{tabular}


Choudhary et al. (2008) sorted wheat into five classes, Teimouri et al. (2016) used the ANN to classify almonds into five different classes. Donis-Gonzalez et al. (2013) classified chestnuts into five classes. However, these authors used colour, texture and shape from the features.

\section{Conclusions}

In this study, design and testing of a machine vision based beans sorter is presented. The proposed system consists of electronic, pneumatic and mechanical parts. The sorting algorithm was based on ANN. After training and validating the network in the offline mode using the NeuroSolution software, the correct classification rate for pinto, white, red, damaged beans and stones were obtained 86.26, 100, 100, 54.9 and $65.3 \%$, respectively. For the four types of beans and stones, the average accuracy of the model was $81.2 \%$. The correct value of classification in the offline mode using NeuroSolutions software for pinto bean and other materials was $97 \%$ and $86.6 \%$, respectively. Finally, the system was evaluated in online mode. For this purpose, 400 beans were placed one by one on the conveyor belt. The overall accuracy of the device was $95.6 \%$, which indicates a good performance of the sorting system.

\section{References}

Almaliki, S., Alimardani, R. and Omid, M. (2016) 'Artificial neural network based modeling of tractor performance at different field conditions', Agricultural Engineering International: CIGR Journal, Vol. 18, No. 4, pp.262-274.

Arboleda, E.R., Fajardo, A.C. and Medina, R.P. (2018) 'Classification of coffee bean species using image processing, artificial neural network and K nearest neighbors', in 2018 IEEE International Conference on Innovative Research and Development (ICIRD), IEEE, May, pp.1-5.

Chen, X., Xunb, Y., Li, W. and Zhanga, J. (2010) 'Combining discriminant analysis and neural networks for corn variety identification', Computers and Electronics in Agriculture, Vol. 71, No. 4, pp.48-53.

De Oliveira, E.M., Leme, D.S., Barbosa, B.H.G., Rodarte, M.P. and Pereira, R.G.F.A. (2016) 'A computer vision system for coffee beans classification based on computational intelligence techniques', Journal of Food Engineering, Vol. 171, No. 4, pp.22-27.

Donis-Gonzalez, I.R., Guyer, D.E., Leiva-Valenzuela, G.A. et al. (2013) 'Assessment of chestnut (Castanea spp.) slice quality using color images', Journal of Food Engineering, Vol. 115, No. 6, pp.407-414.

Ercisli, S., Sayinci, B., Kara, M., Yildiz, C. and Ozturk, I. (2012) 'Determination of size and shape features of walnut (Juglans Regia L.) cultivars using image processing', Scientia Horticulturae, Vol. 133, pp.47-55.

Hong, Y., Lee, W., Chung, H., Lee, H., Son, J., Cho, K. and Park, W. (2002) 'A computer vision system for rice kernel quality evaluation', Annual Meeting, No. 023130.

Jiang, L., Wang, J., Li, Y., Wang, Z., Liang, J., Wang, R. and Zhang, M. (2014) 'Effects of ultrasound on the structure and physical properties of black bean protein isolates', Food Research International, Vol. 62, No. 4, pp.595-601.

Kaliramesh, S., Chelladurai, V., Jayas, D.S., Alagusundaram, K., White, N.D.G. and Fields, P.G. (2013) 'Detection of infestation by Callosobruchus Maculatus in mung bean using near-infrared hyperspectral imaging', Journal of Stored Products Research, Vol. 52, No. 3, pp.107-111. 
Kuo, B.C., Ho, H.H., Li, C.H., Hung, C.C. and Taur, J.S. (2014) 'A kernel-based feature selection method for SVM with RBF kernel for hyperspectral image classification', IEEE Journal of Selected Topics in Applied Earth Observations and Remote Sensing, Vol. 7, No. 1, pp.317-326.

Lorestani, A.N., Omid, M., Bagheri-Shooraki, S., Borghei, A.M. and Tabatabaeefar, A. (2006) 'Design and evaluation of a fuzzy logic based decision support system for grading of golden delicious apples', Int. J. Agric. Biol., Vol. 8, No. 4, pp.440-444.

Mollazade, K., Omid, M. and Arefi, A. (2012) 'Comparing data mining classifiers for grading raisins based on visual features', Computers and Electronics in Agriculture, Vol. 84, No. 9, pp.124-131.

Omid, M., Mahmoudi, A. and Omid, M.H. (2010) 'Development of pistachio sorting system using principal component analysis (PCA) assisted artificial neural network (ANN) of impact acoustics', Expert Systems with Applications, Vol. 37, No. 10, pp.7205-7212.

Piotr, Z. (2011) 'Discrimination of wheat grain varieties using image analysis and neural networks', Journal of Cereal Science, Vol. 54, No. 7, pp.60-68.

Soltani, M., Omid, M. and Alimardani, R. (2015) 'Egg volume prediction using machine vision technique based on pappus theorem and artificial neural network', Journal of Food Science and Technology, Vol. 52, No. 5, pp.3065-3071.

Tadesse, T.F., Gebre, A., Gebremeskel, A.F. and Henry, C.J. (2015) 'Pasting characteristics of starches in flours of chickpea (Cicer Arietinum L.) and faba bean (Vicia Faba L.) as affected by sorting and dehulling practices', African Journal of Food Science, Vol. 9, No. 12, pp.555-559.

Teimouri, N., Omid, M., Mollazade, K. and Rajabipour, A. (2014) 'A novel artificial neural networks assisted segmentation algorithm for discriminating almond nut and shell from background and shadow', Computers and Electronics in Agriculture, Vol. 105, pp.34-43.

Teimouri, N., Omid, M., Mollazade, K. and Rajabipour, A. (2016) 'An artificial neural network-based method to identify five classes of almond according to visual features', Journal of Food Process Engineering, Vol. 39, No. 6, pp.625-635. 